(C) 2005 International Press

Adv. Theor. Math. Phys. 9 (2005) 999-1006

\title{
Seidel's mirror map for the torus
}

\author{
Eric Zaslow \\ Department of Mathematics, Northwestern University, Evanston, \\ IL 60208, USA \\ zaslow@math.northwestern.edu
}

\begin{abstract}
Using only the Fukaya category and the monodromy around large complex structure, we reconstruct the mirror map in the case of a symplectic torus. This realizes an idea described by Paul Seidel.
\end{abstract}

\section{Introduction}

Paul Seidel had the following idea for recovering the mirror map purely from the Fukaya category. ${ }^{1}$ Start with a symplectic Calabi-Yau $X$ and its family of complex structures, and assume it has a projective mirror manifold $Y$ with a family of symplectic structures, and that Kontsevich's conjecture holds: $D F u k(X) \cong D(Y)$, where $D F u k(X)$ is the Fukaya category of $X$ (i.e., the bounded derived category constructed from the Fukaya $A_{\infty}$ category) and $D(Y)$ is the bounded derived category of coherent sheaves on $Y$. Then the homogeneous coordinate ring on $Y$ is given by

$$
\mathcal{R}=\bigoplus_{k=0}^{\infty} \Gamma\left(\mathcal{O}_{Y}(k)\right)=\bigoplus_{k=0}^{\infty} \operatorname{Hom}_{D \operatorname{Fuk}(X)}(\psi(\mathcal{O}), \psi(\mathcal{O}(k))),
$$

e-print archive: http://lanl.arXiv.org/abs/math.SG/0506359

${ }^{1}$ The idea described was told in a private communication to the author. This may have been implicit in the works of Fukaya and/or in the minds of others in the field, and has recently been described by $\mathrm{R}$. Thomas in [11]. 
where $\psi$ is the equivalence of categories. The term on the right can be evaluated solely in $D F u k(X)$, and thus the complex projective variety $Y$ can be recovered. The dependence of this construction on the symplectic structure of $X$ defines the mirror map. ${ }^{2}$

Let $S \equiv \psi(\mathcal{O})$ be the object dual to the structure sheaf of $Y$, conjecturally the Lagrangian section of the Lagrangian torus fibration (cf. [9]). We will often equate a geometric Lagrangian submanifold with the object in DFuk which it defines, including, if necessary, additional data such as grading and local system. Recall [3] that on the complex structure moduli space of $X$, monodromies act by symplectomorphisms, which define autoequivalences of $D F u k(X)$ (we use the same notation for a symplectomorphism and the autoequivalence it induces) and that the monodromy $\rho$ around the large complex structure limit point is mirror to the autoequivalence of $D(Y)$ defined by $\mathcal{E} \rightarrow \mathcal{E} \otimes \mathcal{O}(1)$. We define $L_{k}$ by $L_{k} \equiv \rho^{k} S$. Note $S=L_{0}$ and $L \equiv L_{1}$ is dual to $\mathcal{O}(1)$. In fact, $L_{k}=\psi(\mathcal{O}(k))$, so we wish to compute $\bigoplus_{i} \operatorname{Hom}_{D \operatorname{Fuk}(X)}\left(S, L_{k}\right)$. To interpret this as a ring, we must identify $\operatorname{Hom}\left(L_{k}, L_{k+l}\right)$ with $\operatorname{Hom}\left(S, L_{l}\right)$ (we hereafter drop the $D F u k(X)$ subscript), and to do so we use the symplectomorphism $\rho^{-k}$.

In this note we will compute $\mathcal{R}$ in the case where $X$ is a symplectic two-torus and derive the mirror map. ${ }^{3}$ Without knowing the mirror map, we can still say that $Y$ is some elliptic curve and thus has a projective embedding as a cubic curve. Then $\mathcal{O}(1)$ is a line bundle of degree three on $Y$, so its mirror must have intersection three with $S$. Taking the base section $S$ to be the $x$-axis in the universal cover $\mathbb{R}^{2}$, we have that $L$ is a line of slope three. So we put $\rho=\gamma^{3}$, where $\gamma$ is a minimal Dehn twist, and note that $\rho$ is maximally unipotent. For simplicity, we take $S$ (and therefore $L$ ) to have trivial local systems and to pass through lattice vectors, but our results do not depend on this choice. The data of $S$ and $\rho$ now allows us to calculate $\mathcal{R}$.

\section{Computation}

We define $X=\mathbb{R}^{2} / \mathbb{Z}^{2}$ with $\omega=\tau d x \wedge d y, \tau \in \mathbb{C}, \operatorname{Im}(\tau)>0$. The category constructed from Fukaya's $A_{\infty}$ category in this case was described explicitly in [6-8], and we refer the reader to those papers for details. As discussed above, we have $L_{k}=\left\{(t, 3 k t) \bmod \mathbb{Z}^{2}: t \in \mathbb{R}\right\}$, and we define its

\footnotetext{
${ }^{2}$ The case of Fano varieties is considered in [1].

${ }^{3}$ The result is guaranteed to be correct here, since Kontsevich's conjecture has been proven in this example [8].
} 
grading $\alpha=\tan ^{-1}(k) \in[0, \pi / 2)$. We define $X_{i}=(i / 3,0) \in \operatorname{Hom}(S, L), Y_{i}=$ $(i / 6,0) \in \operatorname{Hom}\left(S, L_{2}\right)$, and $Z_{i}=(i / 9,0) \in \operatorname{Hom}\left(S, L_{3}\right)$, where $i$ is taken $\bmod$ 3,6 , and 9 , respectively. In the sequel, when we write an equation like $X_{1} X_{2}=\ldots$, the $X_{2}$ is understood to live in $\operatorname{Hom}\left(L_{1}, L_{2}\right)$ through $\rho$. Explicitly, $\rho(x, y)=(x, y+3 x)$; indeed $\rho^{*} \omega=\omega$.

Let us compute the products $X_{i} X_{j}$. The Fukaya category for this example was discussed in $[4,8]$. The basic computation is $X_{0} X_{1}$. The minimal triangle (holomorphic map) appearing in the product connects the points $X_{0}=(0,0), \rho\left(X_{1}\right)=X_{1}=(1 / 3,1)$, and $Y_{1}=(1 / 6,0)$ and has symplectic area $(1 / 2)(1 / 6)(1) \tau$. Multiples and translates of this triangle are relevant to other products. Multiples by $6 n$ have the same endpoints and contribute to the same product, with area $(1 / 2)(n+1 / 6)(6 n+1) \tau$. The coefficient of $Y_{1}$ in $X_{0} X_{1}$ is thus $A_{1} \equiv \sum_{n} \exp \left[i \pi 6 \tau(n+1 / 6)^{2}\right]=\theta[1 / 6,0](6 \tau, 0){ }^{4}$ Defining $A_{k}:=\theta[k / 6,0](6 \tau, 0), k \in \mathbb{Z} / 6 \mathbb{Z}$, and noting $A_{k}=A_{6-k}$, we get the following relations:

$$
X_{i} X_{j}=\sum_{k=0}^{1} A_{i-j+3 k} Y_{i+j+3 k} .
$$

The right hand side of this equation makes sense with $i, j$ defined mod 3 . Commutativity is easily shown to follow from the relations among the $A_{k}$.

Next we compute $Y_{i} X_{j}$. Starting with $Y_{1} X_{1}$, the minimal triangle has vertices $Y_{1}=(1 / 6,0), \rho^{2}\left(X_{1}\right)=X_{1}=(1 / 3,1)$, and $Z_{2}=(2 / 9,0)$, with area $(1 / 2)(1 / 18)(1) \tau$. Odd multiples (with left endpoint fixed) and translates of this triangle are relevant to $Y_{i} X_{j}$ with $i$ odd; even multiples and translates to $i$ even. Multiples by $18 n$ have the same endpoints. Therefore $Y_{1} X_{1}=$ $B_{1} Z_{2}+B_{7} Z_{5}+B_{13} Z_{8}$, where $B_{k}=\sum_{n} \exp \left[i \pi 18 \tau(n+k / 18)^{2}\right]=\theta[k / 18,0]$ $(18 \tau, 0)$. Note $B_{k}=B_{18-k}$ and $k$ is defined $\bmod 18$. As an example of another product, the third multiple of the minimal triangle has endpoints $Y_{1}=$ $(1 / 6,0), X_{2}=(2 / 3,3), Z_{3}=(1 / 3,0)$, thus $Y_{1} X_{2}=B_{3} Z_{3}+\cdots$ Collecting results, we find

$$
Y_{i} X_{j}=\sum_{k=0}^{2} B_{2 j-i+6 k} Z_{i+j+3 k} .
$$

\section{Commutativity and associativity}

Associativity in the (derived or cohomological) Fukaya category follows from general grounds, and in the case of the torus amounts to an equality obtained from expressing the area of a non-convex quadrangle by splitting it into

\footnotetext{
${ }^{4}$ We recall the definition $\theta[a, b](\tau, z)=\sum_{n \in \mathbb{Z}} \exp \left[i \pi \tau(n+a)^{2}+2 \pi i(n+a)(z+b)\right]$.
} 
triangles in two different ways. (This was noted, for example, in Section 2 of [6].) It also amounts to relations among the $A_{k}$ and $B_{k}$, which we describe presently.

As for commutativity, this follows from the existence of a robust family of anti-symplectomorphisms. ${ }^{5}$ For example, in considering the products $X_{0} Y_{k}$, one must count (among other things) triangles with vertices $X_{0}, \rho\left(Y_{k}\right)$, and $Z_{k}$ arranged in clockwise orientation and with sides of appropriate slope. Now consider the map $\varphi$ :

$$
(x, y) \longmapsto\left(\frac{1}{2} x-\frac{7}{18} y+\frac{1}{9} k,-2 y\right) .
$$

We note $\varphi\left(X_{0}\right)=Z_{k}, \varphi\left(\rho\left(Y_{k}\right)\right)=X_{0}=\rho^{2}\left(X_{0}\right)$, and $\varphi\left(Z_{k}\right)=Y_{k}$. Further, since $\varphi$ is an anti-symplectomorphism, i.e., $\varphi^{*} \omega=-\omega$, it preserves areas and reverses the orientation and thus changes the order in which the vertices appear on the outside of the triangle. Thus $Y_{k}, \rho^{2}\left(X_{0}\right), Z_{k}$ are oriented clockwise in the image triangle, which has the same area as the original. This proves commutativity among products $X_{0} Y_{k}$. Translations of $\varphi$ suffice for proving commutativity for $X_{j} Y_{k}$. Products $X_{i} X_{j}$ were already seen to be commutative, and this is all that we will require for our purposes. In short, commutativity follows from anti-symplectomorphisms mapping vertices $\left(X, \rho^{n} Y, Z\right)$ to $\left(Z, \rho^{m} X, Y\right)$ in holomorphic triangles. It is not clear (to the author) why commutativity should hold in a general symplectic manifold.

We now return to an explicit description of the associativity constraint. We will make use of the following identity, which follows from the addition formula II.6.4 of [5]:

$$
\begin{aligned}
\theta\left[\frac{a}{n}, 0\right](n \tau, 0) \theta\left[\frac{b}{n k}, 0\right](n k \tau, 0)= & \sum_{\epsilon=0}^{k} \theta\left[\frac{b-k a+k n \epsilon}{k(k+1) n}, 0\right](k(k+1) n \tau, 0) \theta \\
& \times\left[\frac{a+b+k n \epsilon}{(k+1) n}, 0\right]((k+1) n \tau, 0) .
\end{aligned}
$$

When $n=6$ and $k=3$ this gives us formulas for $A_{a} B_{b}$. Defining $C_{c}=$ $\theta[c / 24,0](24 \tau)$ and $D_{d}=\theta[d / 72,0](72 \tau)$, we have

$$
A_{a} B_{b}=\sum_{\epsilon=0}^{3} C_{a+b+18 \epsilon} D_{b-3 a+18 \epsilon} .
$$

\footnotetext{
${ }^{5}$ An example of a noncommutative coordinate ring appears in [10].
} 
This formula suffices for proving some of the equivalences necessary for showing associativity. Others follow from further application of equation (3.1).

For example, one wants to show that $\left(X_{0}^{2}\right) X_{1}=X_{0}\left(X_{0} X_{1}\right)$. This amounts to $\left(A_{0} Y_{0}+A_{3} Y_{3}\right) X_{1}=X_{0}\left(A_{1} Y_{1}+A_{2} Y_{4}\right)$. Using commutativity and the products $(2.1)$, then equating coefficients on $Z_{k}$, gives the conditions

$$
\begin{aligned}
& A_{0} B_{2}+A_{3} B_{7}=A_{1} B_{1}+A_{2} B_{8}, \\
& A_{0} B_{8}+A_{3} B_{1}=A_{1} B_{5}+A_{2} B_{4}, \\
& A_{0} B_{4}+A_{3} B_{5}=A_{1} B_{7}+A_{2} B_{2} .
\end{aligned}
$$

The first and third relations follow immediately from equation (3.2). The second equation is most easily seen by rewriting the right hand side as $A_{-1} B_{5}+A_{-2} B_{-4}$. Proceeding in this manner, one can prove well-definedness of $X_{i} X_{j} X_{k}$.

Again, associativity follows from quadrilateral dissection, or on general grounds for the Fukaya category, and our philosophy here should be to think of these identities as following from the associativity constraints. In either case, we will use the explicit expressions derived here.

\section{Relations}

One finds that the number of degree two polynomials in the three variables $X_{i}$ equals exactly the number of $Y_{k}$, and in fact since $A_{0} A_{1}-A_{2} A_{3} \neq 0$ one finds that the $Y_{k}$ can be written in terms of products $X_{i} X_{j}$, and vice versa, so there are no relations in $\mathcal{R}$ at this degree. At the next level, we have ten independent polynomials and nine $Z_{k}$, so we expect a single relation. Let us search for this relation.

Let

$$
\left\{X_{0}^{3}, X_{1}^{3}, X_{2}^{3}, X_{0}^{2} X_{1}, X_{1}^{2} X_{2}, X_{2}^{2} X_{0}, X_{0}^{2} X_{2}, X_{1}^{2} X_{0}, X_{2}^{2} X_{1}, X_{0} X_{1} X_{2}\right\}
$$

be a basis, with $e^{I}$ the $I$-th entry, $I=0 \cdots 9$. Using the product, we can write $e^{I}=\sum_{k} M_{k}^{I} Z_{k}$. A relation $a$ has the form $\sum_{I} a_{I} e^{I}=0$, or $\sum_{k}\left(\sum_{I}\left(M_{k}{ }^{I} a_{I}\right)\right)$ $Z_{k}=0$. Since the $Z_{k}$ are linearly independent generators of $\operatorname{Hom}\left(S, L_{3}\right)$ we have, in matrix form $M \cdot a=0$, or $a \in \operatorname{Ker}(M)$. $M$ is a $9 \times 10$ matrix, so the kernel should be one-dimensional, and we can take $a_{I}=c(-1)^{I} \operatorname{det}\left(M_{I}\right)$, where $M_{I}$ is $M$ with the $I$-th column removed and $c \neq 0$ is any constant. 
Using the products found in Section 2, one finds

$$
M=\left(\begin{array}{llllllllll}
p & q & q & 0 & 0 & 0 & 0 & 0 & 0 & u \\
0 & 0 & 0 & r & t & s & 0 & 0 & 0 & 0 \\
0 & 0 & 0 & 0 & 0 & 0 & t & r & s & 0 \\
q & p & q & 0 & 0 & 0 & 0 & 0 & 0 & v \\
0 & 0 & 0 & s & r & t & 0 & 0 & 0 & 0 \\
0 & 0 & 0 & 0 & 0 & 0 & s & t & r & 0 \\
q & q & p & 0 & 0 & 0 & 0 & 0 & 0 & v \\
0 & 0 & 0 & t & s & r & 0 & 0 & 0 & 0 \\
0 & 0 & 0 & 0 & 0 & 0 & r & s & t & 0
\end{array}\right)
$$

where

$$
\begin{array}{ccc}
p=A_{0} B_{0}+A_{3} B_{9} & r=A_{0} B_{2}+A_{3} B_{7} & u=A_{2} B_{0}+A_{1} B_{9} \\
q=A_{0} B_{6}+A_{3} B_{3} & s=A_{0} B_{8}+A_{3} B_{1} & v=A_{2} B_{6}+A_{1} B_{3} \\
& t=A_{0} B_{4}+A_{3} B_{5}
\end{array}
$$

Up to a common multiple, one finds $a \sim((p+q) u-2 q v, p v-q u, p v-q u$, $\left.0,0,0,0,0,0,2 q^{2}-p q-p^{2}\right)$. In fact, $u=v$, which follows from associativity, or equivalently the relation (3.1), so we can remove the common (non-zero) factor of $p-q$ and take

$$
a=(u, u, u, 0,0,0,0,0,0,-2 q-p) .
$$

If there are no other relations in the ring $\mathcal{R}$, then this single relation defines a cubic curve in the Hesse family as

$$
a_{0} X_{0}^{3}+a_{1} X_{1}^{3}+a_{2} X_{2}^{3}+a_{9} X_{0} X_{1} X_{2}=0 .
$$

The modular invariant is easily calculated in terms of $z=-(1 / 3) a_{9}$ $\left(a_{0} a_{1} a_{2}\right)^{-1 / 3}=((2 q+p) / 3 u)$. Explicitly,

$$
j(\tau)=-27 z^{3}\left(z^{3}+8\right)^{3}\left(1-z^{3}\right)^{-3} .
$$

This equation, which should define the $j$-function of the mirror curve, is written in terms of the symplectic parameter $\tau$ on the torus. It therefore defines the mirror map, which in this example is known to send the symplectic parameter $\tau$ to the modular parameter $\tau$ in the upper halfplane. So equation (4.1) amounts to an identity in terms of the variable $\tau$, or more conveniently for us, $x=e^{-i \pi \tau / 18}$, and it remains to verify this relation. ${ }^{6}$

\footnotetext{
${ }^{6}$ We ignore the possibility of further relations in $\mathcal{R}$. This assumption is justified using the mirror equivalence but would be difficult to show working purely from the Fukaya side.
} 
The following identities follow directly from the definitions:

$$
\begin{aligned}
& A_{k}=x^{3 k^{2}}+\sum_{n=1}^{\infty} x^{3(6 n+k)^{2}}+x^{3(6 n-k)^{2}} \\
& B_{k}=x^{k^{2}}+\sum_{n=1}^{\infty} x^{(18 n+k)^{2}}+x^{(18 n-k)^{2}} .
\end{aligned}
$$

Recall that the $j$-invariant has the expansion

$$
j(x)=x^{-36}+744+196884 x^{36}+21493760 x^{72}+864299970 x^{108}+\cdots
$$

These coefficients and more can be corroborated order by order in the series expansion of the right hand side of equation (4.1). A more general proof may be found in [2]. Of course, this had to be true, by the equivalence of categories already proved in [8], but our intent was to find this result working only from the Fukaya category. ${ }^{7}$ We find the computation a pleasant realization of Seidel's idea.

\section{Acknowledgments}

I would like to thank Paul Seidel for communicating his ideas freely. Thanks to The Fields Institute for hosting me during this project. This work was supported in part by a Clay Senior Scholars fellowship and by NSF grant DMS-0405859.

\section{References}

[1] M. Abouzaid, Homogeneous Coordinate Rings and Mirror Symmetry for Toric Varieties, math.SG/0511644.

[2] C. Birkenhake and H. Lange, Cubic theta relations, J. Reine Angew. Math. 407 (1990), 167-177.

[3] This was originally proposed by M. Kontsevich in a lecture at Rutgers University, November 11, 1996, and later investigated by R. P. Horja in Hypergeometric functions and mirror symmetry in toric varieties, math.AG/9912109, and by R. Thomas and P. Seidel in Braid group actions on derived categories of coherent sheaves, Duke Math J. 108 (2001), 37-108.

\footnotetext{
${ }^{7}$ Perhaps one could invert this philosophy and derive information about the Fukaya category from the known mirror maps, in cases where computing products is formidable.
} 
[4] M. Kontsevich, Homological algebra of mirror symmetry, in Proceedings of the International Congress of Mathematicians, Vol. 1, 2, (Zürich, 1994), Birkhuser (1995), 120-139.

[5] D. Mumford, Tata lectures of theta I, Birkhäuser, Boston, 1993.

[6] A. Polishchuk, Massey and Fukaya products on elliptic curves, Ad. Theoret. Mathe. Phys. 4 (2000), 1187-1207; math.AG/9803017.

[7] A. Polishchuk, $A_{\infty}$ structures on an elliptic curve, Commun. Math. Phys. 247 (2004), 527-551.

[8] A. Polishchuk and E. Zaslow, Categorical Mirror Symmetry: The Elliptic Curve, Adv. Theoret. Math. Phys. 2 (1998), 443-470; math.AG/9801119.

[9] A. Strominger, S.-T. Yau, and E. Zaslow, Mirror symmetry is T-duality, Nuclear Phys. B479 (1996), 243-259; hep-th/9606040.

[10] M. Aldi and E. Zaslow, Seidel's Mirror Map for Abelian Varieties, math.SG/0512229.

[11] R. P. Thomas, The Geometry of Mirror Symmetry, math.AG/0512412. 